\title{
The Ages and Masses of the Stars.
}

\author{
By J. H. Jeans, Sec. R.S.
}

$A^{\mathrm{S}}$ $\mathrm{S}$ is well-known, a rapidly moving electron has a greater mass than a stationary one; more force is required to deflect it from its path or to produce a specified acceleration. The theory of relativity shows that this is not an isolated phenomenon, but an example of a universal principle. Every natural system, on acquiring additional energy $E$ increases its mass by $E / C^{2}$, and a loss of energy causes a corresponding loss of mass. ${ }^{1}$ For example the sun, losing energy by radiation at the rate of $3.8 \times 10^{33}$ ergs per second, must be losing mass at the rate of $4.2 \times 10^{12}$ grammes (about four million tons) a second.

Changes of energy and changes of mass are, then, the same thing: it is immaterial whether we specify the change in terms of energy or of mass. It is a somewhat different question whether absolute mass can be specified as absolute energy or vice versa. For example, setting an electron in motion with a velocity $0.866 C$ doubles its mass, but in what sense does it double its energy? If $m$ was the mass of the electron when at rest, the addition $m$ to its mass has represented an addition $m C^{2}$ of kinetic energy, but did the original mass $m$ also denote energy $m C^{2}$ ?

Clearly an electron at rest must be thought of as having some energy, for it consists of electric charges which have been brought very close to one another in opposition to their mutual repulsions. If an electron is regarded as a negatively charged sphere, its electrostatic energy is $\frac{3}{4} m C^{2}$. To satisfy the conservation of energy when the electron is in motion, there must be further energy $\frac{1}{4} m C^{2}$ of unknown type, ${ }^{2}$ making energy $m C^{2}$ in all. Presumably the whole of this would be set free if the electron could be persuaded to explode and scatter to infinity.

The energy $m C^{2}$ just discussed may be called "subelectronic " energy, any further energy which electrons may have in virtue of their motion or positions in space being "super-electronic" energy. The two energies become equal when an electron moves with a velocity $0.866 C$, corresponding to a temperature of $4,000,000,000^{\circ}$ Centigrade; for an atom the corresponding temperature is thousands of times higher. In all actual systems, then, the super-electronic energy is insignificant in comparison with the sub-electronic. In the sun the ratio of the two kinds of energies is probably at most of the order of one to a million, and the same is of course true of the two kinds of mass. Of the sun's total mass of $2 \times 10^{33}$ grammes, not more than about $2 \times 10^{27}$ grammes can be "super-electronic."

The sun, as we have seen, is radiating mass away at about four million tons per second. The usual estimates of the sun's age have all been based on the supposition that the only mass available for radiation is the "super-electronic" mass. The estimates reached in this way, about twenty million years, have been too short to satisfy either astronomers or geologists. Some years ago I suggested that the source of stellar radiation was to be found in an actual destruction of matter in a star's interior, the mechanism probably being that

${ }^{1}$ Here and throughout $C$ denotes the velocity of light, $3 \times 10^{10} \mathrm{~cm}$. a second.

'Jeans, "Electricity and Magnetism," 4 th edition, $\S 667$. positive and negative electric charges fell together and annihilated one another. The essential novelty in this suggestion was that it made the enormous "subelectronic" mass of a star available for radiation, in addition to the comparatively puny "super-electronic" mass ; thus it immediately extended our estimate of the sun's life by a factor of the order of a million. Whatever else may be thought of this suggestion, it must be admitted that it solves, with a comfortable margin to spare, the age-long problem of the source of the energy of stellar radiation.

Nothing in the suggestion appears to conflict with modern atomic physics. According to classical electrodynamics, the mutual destructiveness of positive and negative charges was such that all the matter of the universe ought long ago to have melted away in a blaze of glory. The quantum-theory has introduced restrictive forces which, in some way unknown to us, keep positive and negative charges apart, but these forces are not unfailing in their action. At times the electron in the hydrogen atom eludes them and drops down from orbit 3 to orbit 2 , at times it drops farther from orbit 2 to orbit $I$; let it sometimes also drop from orbit I to orbit $o$, and what we have been imagining becomes an accomplished fact.

In the form in which we have so far put it, the whole question looks somewhat speculative. It takes on an entirely new complexion in the light of the definite results recently obtained by Eddington (cf. NATURE, May 3I, I924). Both as a matter of theory and of observation, Eddington finds that a star's luminosity is a function, approximately, of its mass alone. All the stars that are about as massive as Sirius have about the same luminosity as Sirius, stars as massive as our sun are about as luminous as our sun, and so on. At present Sirius has 2.5 times the mass of our sun, and $3^{6}$ times the luminosity. Not having an unlimited store of energy it cannot go on radiating at its present rate for ever; its luminosity must eventually decrease, and some day this will only be equal to that of our present sun. When that day comes, Sirius will be only about as massive as our present sun, so that between now and then it must lose 60 per cent. of its present mass. This is enormously more than the total "super-electronic" mass of Sirius, so that we conclude that the main part of its loss of mass will of necessity be "sub-electronic." Further, we know of no normal process by which mass can escape except by radiation, whence we conclude that the diminution of mass is the equivalent of the energy radiated away.

If this be conceded, Eddington's relation between luminosity and mass fixes quite definitely the rate at which stars change both their luminosity and mass. For example, the sun with a mass of $2 \times 10^{33}$ grammes, is radiating away $4.2 \times 10^{12}$ grammes a second. Thus, after $1,500,000$ millions of years its mass will be reduced by ro per cent., so that from Eddington's table, its luminosity will be reduced by about 30 per cent. In the same way we find that the interval on the evolutionary ladder between Sirius and our sun is approximately $6.4 \times 10^{12}$ years. Between a giant sun of ten 
times the mass of our sun and our sun, the interval is $7.5 \times 10^{12}$ years. These two figures do not differ greatly, for a star squanders its mass at a great rate during its highly luminous giant state ; it is the last part of the journey that takes the time. Both figures are of course enormously large in comparison with any estimates heretofore made on the ages of the stars.

If nothing unexpected intervenes in the next $6.4 \times 10^{\mathbf{1 2}}$ years-and it must be admitted that there is time enough for the unforeseen to occur-Sirius will at the end of that period be similar to our present sun. We cannot, however, assert that $6.4 \times 10^{12}$ years ago our sun was in the state of the present Sirius. The oldfashioned astronomer believes that the whole universe was created only some $10^{9}$ or $10^{\mathbf{1 0}}$ years ago, and until the contrary is proved he has a right to his opinion.

The geologist insists that life on our earth must have existed for millions of years because fossil bones occur under deposits which, he estimates, must have taken millions of years to accumulate. A similar method of reasoning is available to the cosmogonist, who has the advantage over the geologist that he can calculate, instead of estimating, his periods of time.

All calculations in cosmogony have hitherto been made on the supposition that stellar masses remained constant. The conception of diminishing masses brings new features into almost every problem of cosmogony. For example, the orbit of a particle about a gravitating mass is no longer an endlessly repeated ellipse ; it is an ellipse of ever-increasing size, the major axis of which varies inversely as the mass of the attracting body. After $1,500,000$ millions of years, when our sun will only have nine-tenths of its present mass, the radius of the earth's orbit will be ten-ninths of its present size and the year will have lengthened to $45^{\mathrm{I}}$ days. The whole universe of stars is expanding for the same reason and in the same way as the solar system. When, if ever, in the past the average star had four times its present mass, the stars must have been 64 times as closely packed as now, and stars must have interfered with their neighbours 64 times as much as they now do. When due allowance has been made for this, it is found that features are shown by the stars which could be produced by the influence of neighbouring stars in periods just about equal to those we have had under consideration, say six or seven millions of millions of years. These features are the fossil bones of cosmogony.
Foremost among such features must be placed the orbits of binary stars. A newly formed binary star has generally a period of a few hours and an almost circular orbit of radius of the order $5 \times 10^{11} \mathrm{~cm}$. Old binaries show much longer periods, orbits which are frequently highly eccentric and of radius much greater than those of new stars. Statistically, the orbits tend towards conformity with what may be called the " equipartition law"-the law which would be obeyed exactly if the binaries were pushed and pulled about by their neighbours for an unlimited time. Calculation shows that the observed partial conformity to this law indicates a knocking about for a period of the order of that just mentioned, say six million million years.

The same period is indicated by the velocities of the stars in space. Statistically, the most massive stars have the lowest velocities, and there is a pronounced tendency to obey an "equipartition law " under which the velocity varies inversely as the square-root of the mass. This would be obeyed perfectly if the stars had influenced one another for ever; the partial extent to which it is obeyed again indicates a period of the order of six million million years.

A somewhat less convincing "fossil-bone" is provided by a study of the ratios of the masses of the two components of binary stars. As a binary gets older, its components become more equal in mass. According to Aitken, ${ }^{3}$ 20 spectroscopic binaries of early type (B to B8) show an average mass-ratio 0.70 , while 7 of late type ( $F$ to $G$ ) show an average mass ratio of 0.89 . Calculation shows that to pass from a mass-ratio 0.70 to one of 0.89 would require a period of 4.5 million million years. The numerical result is entirely satisfactory, but it must be admitted that it is based on scanty material.

Finally, it may be remarked that the extension of the time scale which is now proposed increases enormously the chance of solar systems being formed by tidal action. With a time scale of $10^{9}$ years, we had to think of systems of planets such as our own as being of necessity extremely rare. With the longer timescale and the recognition that our system of stars must have been more closely packed in the past than now, we can think of planetary systems as being, if not quite the normal accompaniment of a sun, at least fairly freely distributed in space.

8 "The Binary Stars," pp. 206, 207.

\section{The Ages of Peat Deposits.}

\section{By Dr. W. H. Pearsall.}

$\mathrm{R}^{\mathrm{B}}$ ECENT work on the Yorkshire peat has raised some very interesting problems as to the ages of various types of peat deposit in the British Isles. One of the striking features of the Pennine peat is the normal absence of any marked differences in composition, such, for example, as the definite forest layers observed in the Scotch deposits by Prof. Lewis. Typical Pennine peat is usually composed entirely of cotton-grass remains, though here and there the remains of heather or birch point to local desiccation, which can usually be traced to drainage. In the
Ingleborough district, Messrs. Cheetham and Burrell find the basal vegetation to differ very slightly from that of the main peat, though rushes are often present. Birch scrub with local pine and oak is also very widely distributed up to $1900 \mathrm{ft}$. The presence of oak is suggestive, since this tree is not known from deposits older than Neolithic, either in England or Scandinavia.

Mr. J. Holmes first pointed out that neolithic flints occurred about the base of the peat, and now further information is accumulating, largely through the efforts of Mr. F. Buckley and Dr. T. W. Woodhead. They

Nก. 2875 , voL. I I 47 